\title{
Oncogene-induced senescence in pituitary adenomas and carcinomas
}

\author{
Krystallenia I. Alexandraki*, ${ }_{1}^{1}$ Mohammed Munayem Khan*, ${ }^{1}$ Harvinder S. Chahal, ${ }^{1}$ \\ Nadezhda S. Dalantaeva, ${ }^{1}$ Giampaolo Trivellin, ${ }^{1}$ Dan M. Berney, ${ }^{2}$ Philippe Caron, ${ }^{3}$ \\ Vera Popovic, ${ }^{4}$ Marija Pfeifer, ${ }^{5}$ Suzanne Jordan, ${ }^{2}$ Márta Korbonits, ${ }^{1}$ Ashley B. Grossman ${ }^{1}$
}

${ }^{1}$ Centre for Endocrinology, William Harvey Research Institute and ${ }^{2}$ Department of Histopathology, Barts and the London School of Medicine, London, U.K.; ${ }^{3}$ Department of Endocrinology and Metabolic diseases, CHU Larrey, Toulouse, France; ${ }^{4}$ Neuroendocrine Unit, Endocrinology Clinic, University Clinical Center, Belgrade, Serbia; ${ }^{5}$ Department of Endocrinology, Diabetes and Metabolic Diseases, University Medical Centre Ljubljana, Ljubljana, Slovenia

* Contributed equally to this work

\begin{abstract}
OBJECTIVE: The model of 'oncogene-induced senescence' (OIS), resulting in cell-proliferation arrest, has recently been suggested as a possible explanation for the non-progression of pituitary tumours to malignancy. The aim of the study was to compare the expression of $\beta$-galactosidase as a molecular marker of OIS, and p21/p16 as additional markers involved in mediating OIS, in pituitary adenomas, carcinomas and normal pituitary tissue. DESIGN: We performed: a) semi-quantitative immunohistochemistry ( $\beta$-galactosidase, $p 16, p 21)$ in 41 pituitary adenomas [(11 GH-secreting, 9 PRL-secreting, 10 ACTH-secreting, 11 non-functioning (NFPAs)], 6 carcinomas (3 multihormonal: PRL/ACTH/GH, PRL/ACTH, PRL/GH/FSH; 1 non-functioning; 2 ACTH-secreting) and 7 normal pituitary tissues; b) quantitative PCR of mRNA (p16 and p21) in 6 GH-secreting, 6 NFPAs and 6 normal pituitary tissues. RESULTS: $\beta$-galactosidase was significantly increased in $\mathrm{GH}$-secreting tumours $(P=0.002)$, NFPAs $(P=0.04)$, macroadenomas $(P=0.03)$ and carcinomas $(P=0.02)$, as compared to normal pituitary tissue. We found that $p 16$ expression was significantly lower in all tumours (both adenomas and carcinomas) probably secondary to reduced transcription, at least for NFPAs; p21 showed a different biological behaviour, implying that p21 and p16 may play different roles in the senescence of each individual type of adenoma. CONCLUSIONS: $\beta$-galactosidase was significantly over-expressed in GHsecreting and NFPAs, and unexpectedly also in carcinomas. We speculate that the senescence pathway, which may explain the rarity of malignant progression to carcinomas in GH-secreting and NFPAs, might not be universal but cell-type specific.
\end{abstract}

Key words: $\beta$-galactosidase, Pituitary adenoma, Pituitary carcinoma, p16, p21, Senescence 


\section{INTRODUCTION}

Pituitary tumours are present at autopsy in about $20 \%$ of the population, while clinically significant tumours are seen much more commonly than previously reported and are currently considered to have a prevalence of around 80 cases/100,000 inhabitants, a four-fold increased prevalence compared to previous estimates. ${ }^{1}$ However, these relatively common neoplasms rarely undergo malignant transformation, this occurring in around $0.2 \%$ or less of all clinical pituitary tumours. ${ }^{2}$ The causative mechanisms which render pituitary tumours almost invariably benign remain elusive; at the molecular level, abnormalities of intracellular signalling pathways have been well characterised, ${ }^{3,4}$ but the fact that these tumours are usually benign in the presence of activation of oncogenic pathways and inactivation of tumour suppressor pathways suggests that an additional intermediate mechanism may act as an on/off switch system to maintain a benign profile. Several mechanisms have been suggested, with increased apoptosis and lack of new vessel formation being the principal ones, 5,6 although such speculations are not fully supported by the current evidence.

Recently, the model of "oncogene-induced senescence" (OIS) has been used to explain proliferative arrest promoting cellular senescence and thus playing a protective role in the non-progression to malignancy. ${ }^{7,8}$ It has been shown that senescence is mediated by oncogenic activity which triggers the activation of different cell-cycle regulators such as p53 and pRb to mediate the senescence pathways: ${ }^{9}$ the influence of a particular oncogene on its downstream effectors increases progressively during tumour development, with senescence being triggered at a point when tumours have already been initiated but have not reached a fully malignant phenotype. ${ }^{10}$ It has been suggested that OIS requires time to develop, following an initial proliferative phase but eventually resulting in a benign tumour with stable growth arrest, as is the case of pituitary adenoma growth. ${ }^{11}$ Senescent cells show altered cell morphology, including increased lysosomal breakage, causing $\beta$-galactosidase (a lysosomal enzyme) to enter into the cytoplasm. ${ }^{10,12,13}$

Previous studies by Melmed's group have suggested that GH-secreting tumours and NFPAs may both be characterised by activation of a senescence pathway, ${ }^{14}$ but that the mechanism differs between these adenomas. ${ }^{14,15}$ Concerning the specific mechanisms mediating senescence, the main families of CDK inhibitors implicated in senescence, acting as tumour suppressors by negatively regulating progression through the G1 and S phases of the cell cycle, are INK4 and Cip/Kip inhibitors. ${ }^{9} 16$ In particular, regarding the pituitary, previous studies have shown that growth hormone (GH)-secreting adenomas express high levels of senescence-associated $\beta$-galactosidase, co-localised with high levels of $\mathrm{p} 21,{ }^{14}$ a CIP/Kip cyclin-dependent kinases inhibitor (CDKI), whilst non-functioning pituitary adenomas (NFPAs) showed low levels of p21,,$^{15}$ implying a different senescence pathway in different pituitary tumours. These results have been validated in experiments using in vivo and in vitro models of cell adenomas with altered PTTG expression. ${ }^{14,15}$ It has also been suggested that the other major class of CDKI, the INK4-related proteins, such as p16, regulate a separate senescence pathway. Hence, both p16 and p21 may separately or collaboratively be involved in pituitary tumorigenesis. ${ }^{16-19}$

The aim of the present study was to compare the expression of a known marker of senescence, $\beta$-galactosidase, along with the expression of the restriction-point cell cycle restraining proteins $\mathrm{p} 16$ (INK4a) and p21 (CIP-1) in all pituitary adenomas (including prolactinomas and $\mathrm{ACTH}$-secreting tumours, which have not been previously studied) and carcinomas compared to normal pituitary tissue, and to determine whether there is evidence implicating OIS in pituitary adenomas which may be lost in carcinomas.

\section{MATERIALS AND METHODS}

Paraffin blocks of human pituitary tissue were recovered from the departmental tissue bank and stored with the permission of the Human Tissue Storage Authority (UK). All investigations were approved by the relevant national Institutional Review Board, and patients undergoing tumour resection provided written consent. We used tissues from 7 normal pituitaries and 41 pituitary adenomas [11 GH-secreting, 9 prolactinomas, $10 \mathrm{ACTH}$-secreting, 11 NFPAs (all null cell adenomas)]. All pituitary 
adenomas were macroadenomas except for $9 \mathrm{ACTH}-$ secreting microadenomas. None of the patients with adenomas had received any other type of treatment before the operation. In addition, we studied 6 pituitary carcinomas: 3 multihormonal (sample 1/patient 1: first operation for PRL-secreting carcinoma but with additional immunostaining positive for ACTH and $\mathrm{GH}$; sample 2/patient 1: the same patient was operated on four months later with immunostaining positive for PRL and ACTH; sample 3/patient 2: second operation 10 years after his operation followed by external radiotherapy, with the tumour immunostaining positive for PRL, GH and FSH), one non-functioning (sample 4/patient 3: third operation, the first operation 12 years ago and the second five years ago), and $2 \mathrm{ACTH}$-secreting (sample 5/patient 4: second operation five years after his first one which was followed by external radiotherapy; sample 6/patient 5 after her first operation). We explored mRNA expression in 6 samples of normal pituitary tissue, 6 NFPAs and $6 \mathrm{GH}$-secreting adenomas, all fresh-frozen on removal. We used two types of normal pituitaries in our studies. For immunostaining controls we used normal pituitaries adjacent to tumour samples to ensure the best comparison for protein staining. Specifically, the normal pituitaries were all part of resection specimens removed at trans-sphenoidal surgery for presumptive tumours which proved to have normal pituitary tissue on histological staining, as previously noted and explained $;{ }^{20}$ specimens of other pituitary pathologies such cysts or lymphocytic hypophysitis were not included in the study. For RNA work we used autopsy samples removed 6-24hrs after death from patients with no known endocrine disease or treatment known to alter pituitary function.

The tissue samples were prepared using routine laboratory techniques, as previously described. ${ }^{21}$ Sections underwent heat-mediated antigen retrieval treatment, before immunohistochemical analysis with the HRP Polymer kit (Launch Diagnostics, Kent, UK). The positive control for $\beta$-galactosidase was human colon tissue section, while that for both p21 and 16 tissue was minimally-invasive cervical cancer. Appropriate negative controls were run alongside each case.

The staining was carried out according to conventional protocols and manufacturers' instructions. For $\beta$-galactosidase expression, a purified rabbit polyclonal antibody raised against the recombinant full length protein raised in E. coli (Abcam ab616, Stratech Scientific Ltd, Newmarket, Suffolk, UK) was used, with a final dilution of 1:500. For p21 expression human WAF1 protein was used (NCL-L-WAF-1, clone 4D10, Leica Microsystems, Newcastle, UK), with a final dilution 1:250. For p16 expression p16INK4a antibody (clone $\mathrm{E} 6 \mathrm{H} 4^{\mathrm{TM}}$, CINtec) was used with a final dilution of 1:30. For Ki67 expression the clone MIB1 was used (Dako Code M7240) with a final dilution of $1 / 200$. All these antibodies were detected using the polymer detection kit (Launch Code HK QD430-XAKE), as per the kit's instructions.

Cytoplasmic staining was classified as diffuse or focal and then scored based on intensity score: strong, moderate, weak, negative, providing a score of: 3,2 , 1 or 0 , respectively, and the extension score: diffuse (considered as value 2) or focal (considered as value 1) score. The final score was the product of the intensity score and extension score using the following formula: Cytoplasmic staining score $=$ intensity score $x$ extension score, with final values therefore ranging from 0 to 6 .

Nuclear staining of cells was classified as following: staining was counted as positive when nuclear staining was strong or moderate, while staining was negative when cells presented weak or no staining. The percentage of positive or negative cells was then expressed as a ratio of positive or negative cells to the total number of cells counted. In each case, sections were assessed by two observers blinded to the diagnosis. In each section, 500 cells were analysed from a randomised grid array.

For 3 groups of fresh-frozen tissue samples ( 6 of normal pituitary tissue, 6 of NFPA and 6 somatotroph tumours), total RNA from tissues was extracted using the RNeasy Mini Kit (Qiagen, Crawley, UK) following the manufacturer's instructions. RNA concentration was assessed by Nanodrop-1000 (Thermo Scientific Wilmington, DE, USA) and RNA integrity was measured by Agilent Bioanalyzer 2100 System (Agilent, Stockport, UK). Only samples with a RNA Integrity Number $(\mathrm{RIN})>7.9$ were further analysed. Expression levels of CDKN2A (p16) and CDKN1A (p21) were assessed by RT-qPCR with the TaqMan system (Applied Biosystems, Foster City, CA, USA) using 
ready-made probe-primer kits (Hs00923894_m1 for CDKN2A, Hs00355782_m1 for CDKN1A). Reactions were performed in triplicate in 384-well plates on a 7900HT Real-Time PCR System (Applied Biosystems) using GAPDH (Hs99999905_m1) as endogenous control. An initial denaturation step of $10 \mathrm{~min}$ at $95^{\circ} \mathrm{C}$ was followed by 40 cycles of $95^{\circ} \mathrm{C}$ for $15 \mathrm{sec}$ and $60^{\circ} \mathrm{C}$ for $10 \mathrm{sec}$. Data were analysed with a relative standard curve using a human reference cDNA as a template (Clontech, Mountain View, CA, USA). The data are expressed as the ratios p16 expression $(\mathrm{g} / \mu \mathrm{l})$-to- GAPDH $(\mathrm{g} / \mu \mathrm{l})$ and $\mathrm{p} 21$ expression $(\mathrm{g} / \mu \mathrm{l})$ -

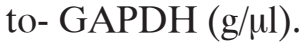

The mitotic index Ki-67 expression of the histological specimens was available in $4 \mathrm{GH}$-secreting adenomas, one prolactinoma, 4 ACTH-secreting, 6 NFPAs and 4 pituitary carcinomas.

Comparisons among the groups for semi-quantitative immunocytochemistry were performed with the Kruskal-Wallis test with Conover-Inman correction and values are presented as median values (range) for multiple comparisons, and the Mann-Whitney U tests for comparisons between two groups. For the quantitative PCR, the data were log-transformed to normalise their distribution and then subjected to a one-way analysis of variance with Newman-Keuls test for multiple comparisons; these values are presented as mean values \pm standard deviation (SD). Statistical significance was accepted at $\mathrm{P}<0.05$. Correlations between variables were evaluated by Spearman's correlation. The analysis was performed using StatsDirect statistical software [Version 2.7.2 (15/9/2008)].

\section{RESULTS}

The staining for $\beta$-galactosidase was cytoplasmic alone (Figures 1a to 1d). In terms of individual comparisons, both GH-secreting and NFPAs as well as the macroadenoma and carcinoma group showed significantly higher $\beta$-galactosidase staining compared to the normal pituitary (Table 1, Figure 2). Furthermore, $\beta$-galactosidase staining was statistically higher in carcinomas and macroadenomas compared to either microadenomas or normal tissue. It should be noted that the only two cases of pituitary carcinoma with $\beta$-galactosidase staining score 6 had previously received external radiotherapy. However, when these two cases were excluded, the carcinomas still did not differ from the adenomas alone.

For p21 and p16, we observed predominantly nuclear (Tables 2,3) but also cytoplasmic (Tables 4,5) staining (Figures 3 and 4). Regarding nuclear p16 staining, this was particularly low in the group of pituitary carcinomas, but all the other individual tumours and the subgroups of adenomas differed compared to normal tissue. Regarding cytoplasmic staining, this was also lower in the carcinomas compared to normal pituitary as well as compared to macroadenomas and lower in micro- amd macroadenomas compared to normal pituitary. The cytoplasmic staining was also found to be very weak or negative in $1 / 6(16.7 \%)$ of normal pituitaries, in 3/9 $(33.3 \%)$ of $\mathrm{GH}$-secreting adenomas, in 1/2 $(50 \%$ ) of PRL-secreting (two were too thickly sectioned to be interpreted), in $1 / 8(12.5 \%)$ of ACTH-secreting, in 4/11 (36.4\%) of NFPAs and in all four $(100 \%)$ pituitary carcinomas. For these rates of negative staining we found differences between normal pituitaries and carcinomas $[\mathrm{P}=0.02$, 95\% CI -0.97- (-0.18)]. Quantitative PCR showed that in the NFPAs $(5.47 \pm 11.7)$ p16 expression was lower $(\mathrm{P}=0.05)$ compared to the normal pituitary (11.17 \pm 7.7$)$; in $\mathrm{GH}$-secreting tumours p16 expression $(8.54 \pm 7.2)$ was still lower but did not quite attain statistical significance $(\mathrm{P}=0.6)$. Furthermore, p16 mRNA expression in NFPAs was less compared to the $\mathrm{GH}$-secreting tumours, but only approaching statistical significance $(\mathrm{P}=0.056)$.

In terms of p21, differences were noted only among individual tumours: p21 nuclear staining was higher in GH-secreting compared to NFPAs or prolactin-secreting tumours. Regarding the analysis for the few specimens with cytoplasmic staining, it was found to be very weak or negative in 6/7 (85.7\%) normal pituitaries, in all $10(100 \%) \mathrm{GH}$-secreting adenomas, in 3/5 (60\%) of PRL-secreting (one too thickly sectioned to be interpreted), in $8 / 9$ (88.9\%) ACTH-secreting, in 4/10 (40\%) of NFPAs and in 4/5 $(80 \%)$ of the pituitary carcinomas. For these rates of negative staining, there were differences between GH-secreting adenomas and NFPAs $(\mathrm{P}=0.005,95 \%$ CI 0.24-0.84), GH-secreting versus PRL-secreting adenomas $(\mathrm{P}=0.02,95 \% \mathrm{CI} 0.12-0.82)$, and NFPAs versus $\mathrm{ACTH}$-secreting adenomas $(\mathrm{P}=0.03,95 \% \mathrm{CI}$ 0.05-0.77). Quantitative PCR showed that $\mathrm{p} 21$ expression in NFPAs $(1.32 \pm 0.83)$ was significantly lower $(\mathrm{P}=0.04)$ than the normal pituitary $(4.21 \pm 2.84)$, while 


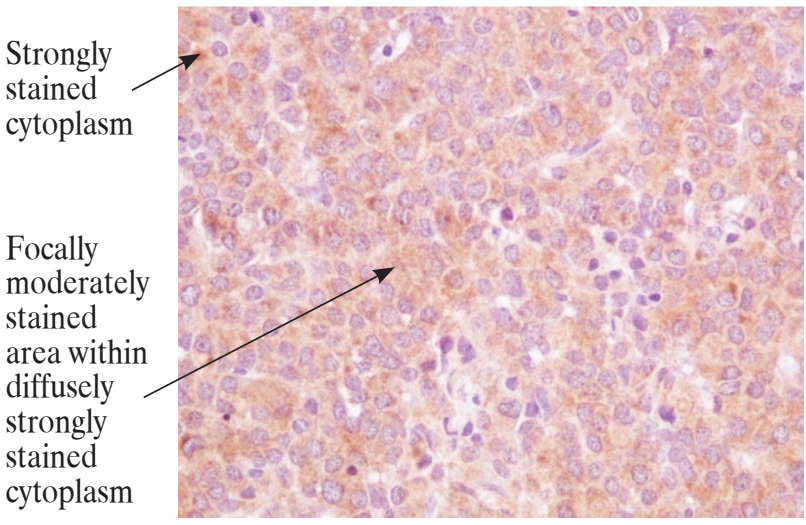

Figure 1A. Examples of cytoplasmic $\beta$-galactosidase immunostaining in pituitary tumours (magnification $\mathrm{x} 1000$ ). GH-secreting adenoma: cytoplasmic staining score $=6$.

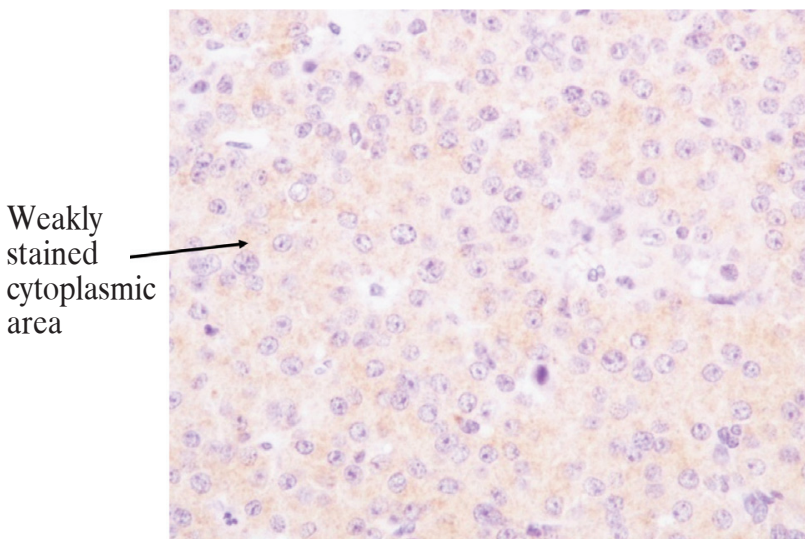

Figure 1C. Examples of cytoplasmic $\beta$-galactosidase immunostaining in normal pituitary (magnification x 1000). Normal pituitary: cytoplasmic staining score $=2$.

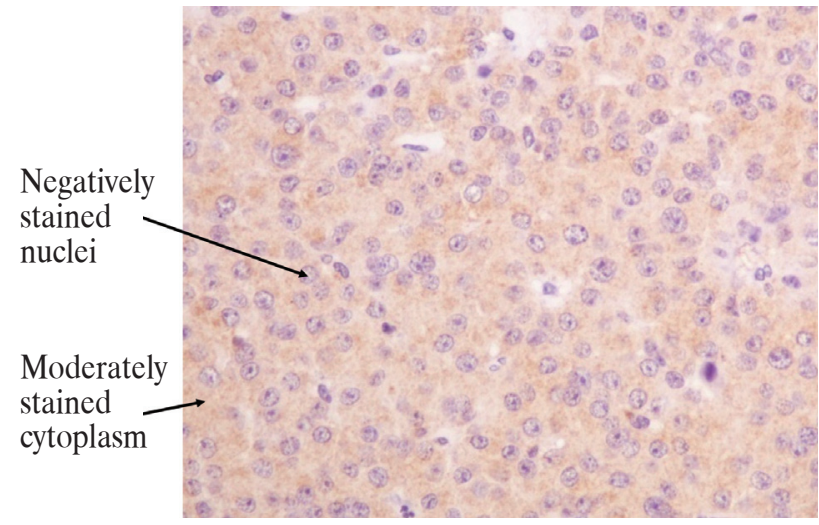

Figure 1B. Examples of cytoplasmic $\beta$-galactosidase immunostaining in pituitary tumours (magnification $\mathrm{x} 1000$ ). Non functioning pituitary adenoma: cytoplasmic staining score $=2$.

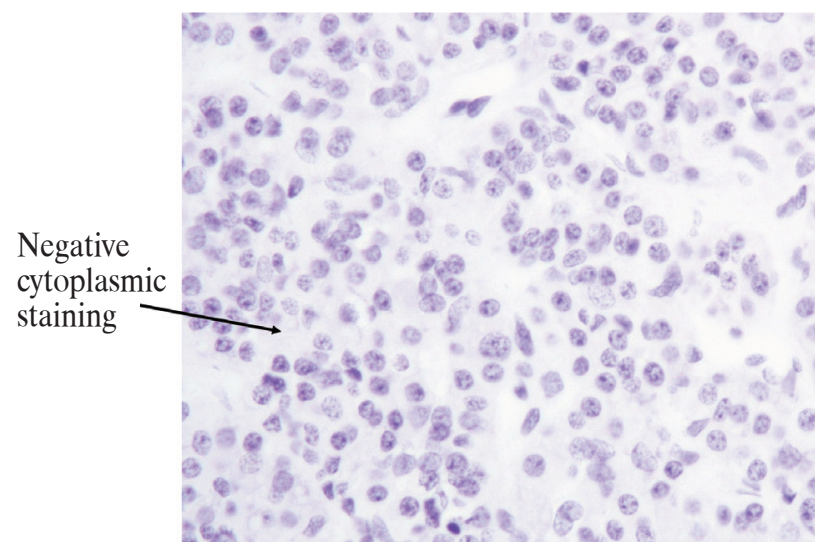

Figure 1D. Examples of cytoplasmic $\beta$-galactosidase immunostaining in normal pituitary (magnification x 1000). Normal pituitary: cytoplasmic staining score $=0$.

Figure 1. Cytoplasmic staining for $\beta$-galactosidase (1A) and both nuclear and cytoplasmic (intensity score) staining for p16 (1B and $1 \mathrm{C}$, respectively) and p21 (1D and 1E, respectively). Mean values and standard deviation were used for a better visual presentation of the results. The statistically significant differences are depicted with lines which connect the groups being compared. NFPA: nonfunctioning pituitary adenomas.

the GH-secreting tumours $(4.25 \pm 2.60)$ were similar to the normal pituitary $(\mathrm{P}=0.98)$ and no statistically significant difference was seen between NFPAs and $\mathrm{GH}$-secreting adenomas $(\mathrm{P}=0.098)$. Thus, the patterns for nuclear and cytoplasmic staining were different for each tumour type.

When all the pituitary adenomas and carcinomas were considered as a single group, the Ki-67 was negatively correlated with the cytoplasmic expression of $\mathrm{p} 16(\mathrm{r}=-0.52, \mathrm{P}=0.03)$ and $\mathrm{p} 21(\mathrm{r}=-0.63, \mathrm{P}=0.004)$, while the cytoplasmic and nuclear expession of p16 were positively correlated with each other $(r=+0.65$,
$\mathrm{P}<0.0001)$. When the pituitary adenomas alone were considered, Ki-67 was negatively correlated with the cytoplasmic expression of $\mathrm{p} 21(\mathrm{r}=-0.63, \mathrm{P}=0.012)$. The cytoplasmic and nuclear expession of $\mathrm{p} 16$ were positively correlated with each other $(\mathrm{r}=+0.59, \mathrm{P}<0.001)$, while there was a trend for cytoplasmic and nuclear expession p21 to be negatively correlated with each other $(r=-0.30, P=0.076)$.

In order to compare the same tissue type, the ACTH-secreting adenomas were compared to carcinomas secreting ACTH: $\beta$-galactosidase staining was found to be higher in the carcinomas at borderline 
TABLE 1. A comparison of the different tumour types with normal pituitary in terms of $\beta$-galactosidase cytoplasmic staining score

\begin{tabular}{|c|c|c|c|c|c|c|}
\hline \multirow[b]{2}{*}{ Pituitary tissue type } & \multirow[b]{2}{*}{ Staining score } & \multicolumn{5}{|c|}{ P value vs. } \\
\hline & & Normal & GH-oma & PRL-oma & ACTH-oma & NFPA \\
\hline Normal $(n=7)$ & $2(0-4)$ & $\mathrm{N} / \mathrm{A}$ & 0.0019 & 0.6832 & 0.8804 & 0.0384 \\
\hline GH-oma (n=11) & $4(2-6)$ & 0.0019 & $\mathrm{~N} / \mathrm{A}$ & 0.0047 & 0.0011 & 0.2157 \\
\hline PRL-oma (n=8) & $2(1-4)$ & 0.6832 & 0.0047 & N/A & 0.7724 & 0.0834 \\
\hline ACTH-oma $(\mathrm{n}=10)$ & $2(0-4)$ & 0.8804 & 0.0011 & 0.7724 & $\mathrm{~N} / \mathrm{A}$ & 0.0342 \\
\hline \multirow[t]{2}{*}{$\operatorname{NFPA}(n=10)$} & $4(2-6)$ & 0.0384 & 0.215 & 0.0834 & 0.0342 & N/A \\
\hline & & Normal & \multicolumn{2}{|c|}{ Adenoma } & \multicolumn{2}{|c|}{ Carcinoma } \\
\hline Normal $(n=7)$ & $2(0-4)$ & N/A & \multicolumn{2}{|c|}{0.1039} & \multicolumn{2}{|c|}{0.017} \\
\hline Adenoma $(\mathrm{n}=39)$ & $4(0-6)$ & 0.1039 & \multicolumn{2}{|c|}{ N/A } & \multicolumn{2}{|c|}{0.1262} \\
\hline \multirow[t]{2}{*}{ Carcinoma $(\mathrm{n}=6)$} & $4(2-6)$ & 0.017 & \multicolumn{2}{|c|}{0.1262} & \multicolumn{2}{|c|}{$\mathrm{N} / \mathrm{A}$} \\
\hline & & Normal & Microadenoma & \multicolumn{2}{|c|}{ Macroadenoma } & arcinoma \\
\hline Normal $(n=7)$ & $2(0-4)$ & $\mathrm{N} / \mathrm{A}$ & 0.8513 & \multicolumn{2}{|c|}{0.0335} & 0.0129 \\
\hline Microadenoma $(\mathrm{n}=11)$ & $2(0-4)$ & 0.8513 & N/A & \multicolumn{2}{|c|}{0.0352} & 0.0142 \\
\hline Macroadenoma $(n=29)$ & $4(1-6)$ & 0.0335 & 0.0352 & \multicolumn{2}{|c|}{ N/A } & 0.2529 \\
\hline Carcinoma $(n=6)$ & $4(2-6)$ & 0.0129 & 0.0142 & \multicolumn{2}{|c|}{0.2529} & $\mathrm{~N} / \mathrm{A}$ \\
\hline
\end{tabular}

Median value (range); comparison between groups was performed using with the Kruskal-Wallis test with Conover-Inman correction; GH-oma: GH-secreting adenoma; PRL-oma: Prolactinoma; ACTH-oma: ACTH-secreting adenoma; NFPA: non functioning pituitary adenoma.
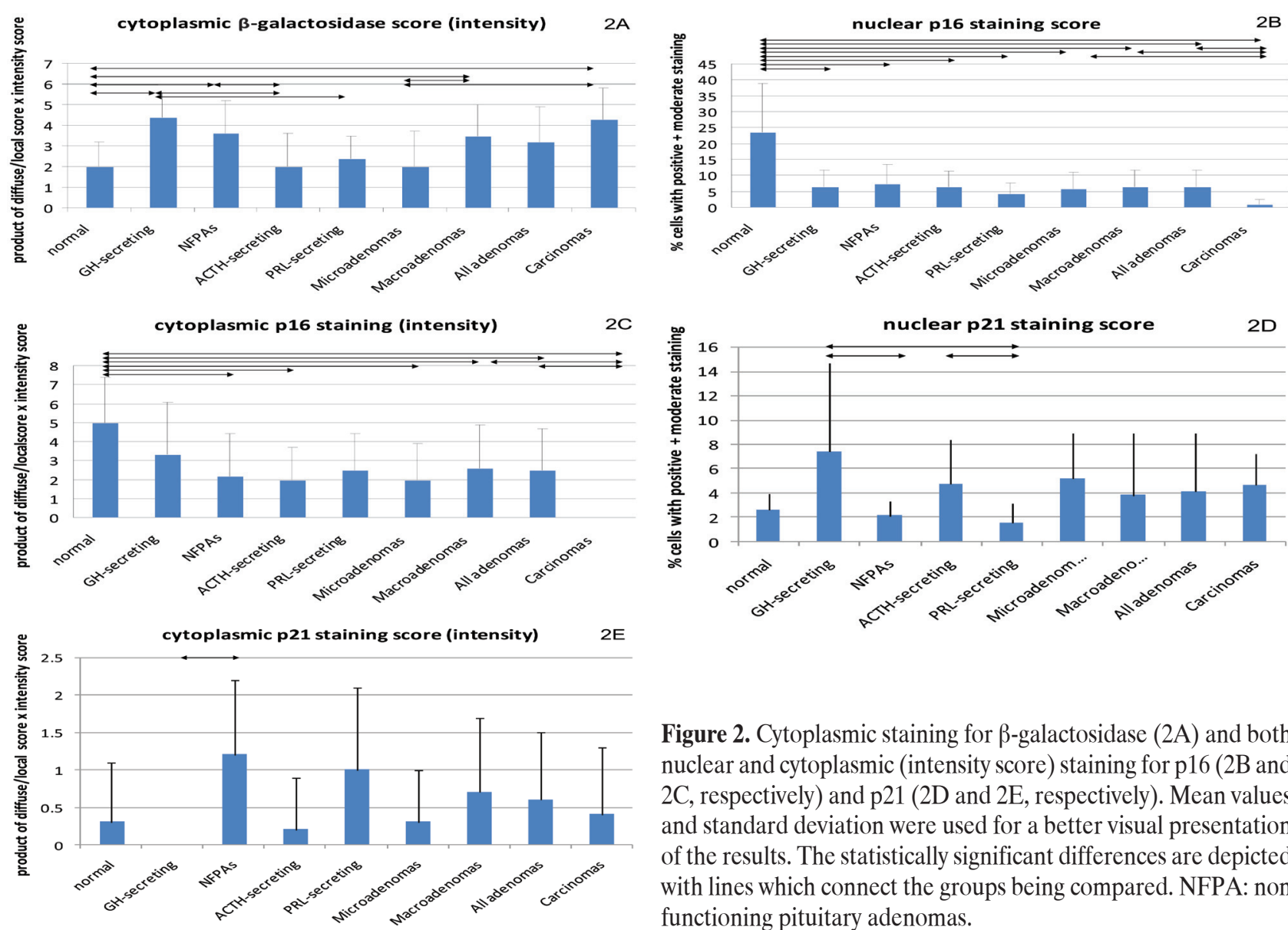

Figure 2. Cytoplasmic staining for $\beta$-galactosidase (2A) and both nuclear and cytoplasmic (intensity score) staining for $\mathrm{p} 16$ (2B and $2 \mathrm{C}$, respectively) and p21 (2D and 2E, respectively). Mean values and standard deviation were used for a better visual presentation of the results. The statistically significant differences are depicted with lines which connect the groups being compared. NFPA: non functioning pituitary adenomas. 
TABLE 2. A comparison of the different tumour types with normal pituitary in terms of p16 nuclear staining score

\begin{tabular}{|c|c|c|c|c|c|c|}
\hline \multirow[b]{2}{*}{ Pituitary tissue type } & \multirow[b]{2}{*}{ Staining score } & \multicolumn{5}{|c|}{$P$ value $v s$. } \\
\hline & & Normal & GH-oma & PRL-oma & ACTH-oma & NFPA \\
\hline Normal $(n=6)$ & $21.1(2.1-47.9)$ & $\mathrm{N} / \mathrm{A}$ & 0.0153 & 0.0071 & 0.0168 & 0.0164 \\
\hline GH-oma $(n=10)$ & $6(0.6-13.5)$ & 0.0153 & $\mathrm{~N} / \mathrm{A}$ & 0.5242 & 0.9883 & 0.9324 \\
\hline PRL-oma $(n=6)$ & $4.3(0.4-7.7)$ & 0.007 & 0.5242 & $\mathrm{~N} / \mathrm{A}$ & 0.541 & 0.4714 \\
\hline ACTH-oma $(n=9)$ & $5.6(0-16)$ & 0.0168 & 0.9883 & 0.541 & $\mathrm{~N} / \mathrm{A}$ & 0.9223 \\
\hline \multirow[t]{2}{*}{$\operatorname{NFPA}(n=11)$} & $5.8(0-19.7)$ & 0.0164 & 0.9324 & 0.4714 & 0.9223 & N/A \\
\hline & & Normal & \multicolumn{2}{|c|}{ Adenoma } & \multicolumn{2}{|c|}{ Carcinoma } \\
\hline Normal $(n=6)$ & $21.1(2.1-47.9)$ & $\mathrm{N} / \mathrm{A}$ & \multicolumn{2}{|c|}{0.0031} & \multicolumn{2}{|c|}{0.0001} \\
\hline Adenoma $(n=36)$ & $5.7(0-19.7)$ & 0.0031 & \multicolumn{2}{|c|}{$\mathrm{N} / \mathrm{A}$} & \multicolumn{2}{|c|}{0.0156} \\
\hline \multirow[t]{2}{*}{ Carcinoma $(n=4)$} & $0.6(0-2.4)$ & 0.0001 & \multicolumn{2}{|c|}{0.0156} & \multicolumn{2}{|c|}{$\mathrm{N} / \mathrm{A}$} \\
\hline & & Normal & Microadenoma & \multicolumn{2}{|c|}{ Macroadenoma } & Carcinoma \\
\hline Normal $(n=6)$ & $21.1(2.1-47.9)$ & $\mathrm{N} / \mathrm{A}$ & 0.0109 & \multicolumn{2}{|c|}{0.0045} & 0.0002 \\
\hline Microadenoma $(n=10)$ & $5.02(0-16.2)$ & 0.0109 & $\mathrm{~N} / \mathrm{A}$ & \multicolumn{2}{|c|}{0.8237} & 0.0489 \\
\hline Macroadenoma $(n=27)$ & $6.1(0-19.7)$ & 0.0045 & 0.8237 & \multicolumn{2}{|c|}{$\mathrm{N} / \mathrm{A}$} & 0.0168 \\
\hline Carcinoma $(n=4)$ & $0.6(0-2.4)$ & 0.0002 & 0.0489 & \multicolumn{2}{|c|}{0.0168} & $\mathrm{~N} / \mathrm{A}$ \\
\hline
\end{tabular}

Median value (range); comparison between groups was performed using with the Kruskal-Wallis test with Conover-Inman correction; GH-oma: GH-secreting adenoma; PRL-oma: Prolactinoma; ACTH-oma: ACTH-secreting adenoma; NFPA: non functioning pituitary adenoma.

TABLE 3. A comparison of the different tumour types with normal pituitary in terms of $\mathrm{p} 21$ nuclear staining score

\begin{tabular}{|c|c|c|c|c|c|c|}
\hline \multirow[b]{2}{*}{ Pituitary tissue type } & \multirow[b]{2}{*}{ Staining score } & \multicolumn{5}{|c|}{$P$ value vs. } \\
\hline & & Normal & GH-oma & PRL-oma & ACTH-oma & NFPA \\
\hline Normal $(n=7)$ & $2.8(0-4.3)$ & $\mathrm{N} / \mathrm{A}$ & 0.1585 & 0.1314 & 0.5741 & 0.4666 \\
\hline GH-oma $(n=10)$ & $4.6(0.9-21.1)$ & 0.1585 & $\mathrm{~N} / \mathrm{A}$ & 0.0035 & 0.3629 & 0.0216 \\
\hline PRL-oma $(\mathrm{n}=7)$ & $1.3(0-4.8)$ & 0.1314 & 0.0035 & $\mathrm{~N} / \mathrm{A}$ & 0.0338 & 0.3549 \\
\hline ACTH-oma $(n=9)$ & $3.3(0.8-9.6)$ & 0.5741 & 0.3629 & 0.0338 & $\mathrm{~N} / \mathrm{A}$ & 0.1665 \\
\hline \multirow[t]{2}{*}{$\operatorname{NFPA}(n=10)$} & $2.1(0.4-3.8)$ & 0.4666 & 0.0216 & 0.3549 & 0.1665 & $\mathrm{~N} / \mathrm{A}$ \\
\hline & & Normal & \multicolumn{2}{|c|}{ Adenoma } & \multicolumn{2}{|c|}{ Carcinoma } \\
\hline Normal $(n=7)$ & $2.8(0-4.3)$ & $\mathrm{N} / \mathrm{A}$ & \multicolumn{2}{|c|}{0.9028} & \multicolumn{2}{|c|}{0.3373} \\
\hline Adenomas $(n=36)$ & $2.4(0-21.1)$ & 0.9028 & \multicolumn{2}{|c|}{$\mathrm{N} / \mathrm{A}$} & \multicolumn{2}{|c|}{0.2725} \\
\hline \multirow[t]{2}{*}{ Carcinomas $(n=6)$} & $5.3(1.3-7.7)$ & 0.3373 & \multicolumn{2}{|c|}{0.2725} & \multicolumn{2}{|c|}{$\mathrm{N} / \mathrm{A}$} \\
\hline & & Normal & Microadenoma & \multicolumn{2}{|c|}{ Macroadenoma } & arcinoma \\
\hline Normal $(n=7)$ & $2.8(0-4.3)$ & $\mathrm{N} / \mathrm{A}$ & 0.3295 & \multicolumn{2}{|c|}{0.8522} & 0.327 \\
\hline Microadenoma $(n=10)$ & $4.8(0.8-9.6)$ & 0.3295 & $\mathrm{~N} / \mathrm{A}$ & \multicolumn{2}{|c|}{0.1485} & 0.9397 \\
\hline Macroadenoma $(n=27)$ & $2.1(0-21.1)$ & 0.8522 & 0.1485 & \multicolumn{2}{|c|}{$\mathrm{N} / \mathrm{A}$} & 0.168 \\
\hline Carcinoma $(n=6)$ & $5.3(1.3-7.7)$ & 0.327 & 0.9397 & \multicolumn{2}{|c|}{0.168} & $\mathrm{~N} / \mathrm{A}$ \\
\hline
\end{tabular}

Median value (range); comparison between groups was performed using with the Kruskal-Wallis test with Conover-Inman correction; GH-oma: GH-secreting adenoma; PRL-oma: Prolactinoma; ACTH-oma: ACTH-secreting adenoma; NFPA: non functioning pituitary adenoma. 
TABLE 4. A comparison of the different tumour types with normal pituitary in terms of p16 cytoplasmic staining score

\begin{tabular}{|c|c|c|c|c|c|c|}
\hline \multirow[b]{2}{*}{ Pituitary tissue type } & \multirow[b]{2}{*}{ Staining score } & \multicolumn{5}{|c|}{$P$ value $v s$. } \\
\hline & & Normal & GH-oma & PRL-oma & ACTH-oma & NFPA \\
\hline Normal $(n=7)$ & $6(0-6)$ & $\mathrm{N} / \mathrm{A}$ & 0.2258 & 0.1669 & 0.0403 & 0.0457 \\
\hline GH-oma $(n=9)$ & $4(0-6)$ & 0.2258 & $\mathrm{~N} / \mathrm{A}$ & 0.6661 & 0.3224 & 0.3771 \\
\hline PRL-oma $(n=4)$ & $3(0-4)$ & 0.1669 & 0.6661 & $\mathrm{~N} / \mathrm{A}$ & 0.7267 & 0.8111 \\
\hline ACTH-oma $(n=9)$ & $2(0-6)$ & 0.0403 & 0.3224 & 0.7267 & $\mathrm{~N} / \mathrm{A}$ & 0.8752 \\
\hline \multirow[t]{2}{*}{$\operatorname{NFPA}(n=11)$} & $2(0-6)$ & 0.0457 & 0.3771 & 0.8111 & 0.8752 & $\mathrm{~N} / \mathrm{A}$ \\
\hline & & Normal & \multicolumn{2}{|c|}{ Adenomas } & \multicolumn{2}{|c|}{ Carcinoma } \\
\hline Normal $(n=7)$ & $6(0-6)$ & $\mathrm{N} / \mathrm{A}$ & \multicolumn{2}{|c|}{0.0423} & \multicolumn{2}{|c|}{0.0016} \\
\hline Adenomas $(n=33)$ & $2(0-6)$ & 0.0423 & \multicolumn{2}{|c|}{$\mathrm{N} / \mathrm{A}$} & \multicolumn{2}{|c|}{0.0233} \\
\hline \multirow[t]{2}{*}{ Carcinomas $(n=4)$} & 0 & 0.0016 & \multicolumn{2}{|c|}{0.0233} & \multicolumn{2}{|c|}{$\mathrm{N} / \mathrm{A}$} \\
\hline & & Normal & Microadenoma & \multicolumn{2}{|c|}{ Macroadenoma } & Carcinoma \\
\hline Normal $(n=7)$ & $6(0-6)$ & $\mathrm{N} / \mathrm{A}$ & 0.0458 & \multicolumn{2}{|c|}{0.0579} & 0.0019 \\
\hline Microadenoma $(n=11)$ & $2(0-6)$ & 0.0458 & $\mathrm{~N} / \mathrm{A}$ & \multicolumn{2}{|c|}{0.5816} & 0.1004 \\
\hline Macroadenoma $(n=28)$ & $2(0-6)$ & 0.0579 & 0.5816 & \multicolumn{2}{|c|}{$\mathrm{N} / \mathrm{A}$} & 0.0249 \\
\hline Carcinoma $(n=4)$ & 0 & 0.0019 & 0.1004 & \multicolumn{2}{|c|}{0.0249} & $\mathrm{~N} / \mathrm{A}$ \\
\hline
\end{tabular}

Median value (range); comparison between groups was performed using with the Kruskal-Wallis test with Conover-Inman correction; GH-oma: GH-secreting adenoma; PRL-oma: Prolactinoma; ACTH-oma: ACTH-secreting adenoma; NFPA: non functioning pituitary adenoma.

TABLE 5. A comparison of the different tumour types with normal pituitary in terms of p21 cytoplasmic staining score

\begin{tabular}{|c|c|c|c|c|c|c|}
\hline \multirow[b]{2}{*}{ Pituitary tissue type } & \multirow[b]{2}{*}{ Staining score } & \multicolumn{5}{|c|}{ P value vs. } \\
\hline & & Normal & GH-oma & PRL-oma & ACTH-oma & NFPA \\
\hline Normal $(\mathrm{n}=7)$ & $0(0-2)$ & $\mathrm{N} / \mathrm{A}$ & 0.6065 & 0.2573 & 0.9107 & 0.1049 \\
\hline GH-oma $(n=10)$ & 0 & 0.6065 & $\mathrm{~N} / \mathrm{A}$ & 0.091 & 0.6672 & 0.0213 \\
\hline PRL-oma $(n=6)$ & $1(0-2)$ & 0.2573 & 0.091 & $\mathrm{~N} / \mathrm{A}$ & 0.1941 & 0.7305 \\
\hline ACTH-oma $(n=9)$ & $0(0-2)$ & 0.9107 & 0.6672 & 0.1941 & $\mathrm{~N} / \mathrm{A}$ & 0.0643 \\
\hline \multirow[t]{2}{*}{$\operatorname{NFPA}(n=10)$} & $2(0-2)$ & 0.1049 & 0.0213 & 0.7305 & 0.0643 & $\mathrm{~N} / \mathrm{A}$ \\
\hline & & Normal & \multicolumn{2}{|c|}{ Adenoma } & \multicolumn{2}{|c|}{ Carcinoma } \\
\hline Normal $(n=7)$ & $0(0-2)$ & $\mathrm{N} / \mathrm{A}$ & \multicolumn{2}{|c|}{0.5643} & \multicolumn{2}{|c|}{0.8702} \\
\hline Adenomas $(\mathrm{n}=35)$ & $0(0-2)$ & 0.5643 & \multicolumn{2}{|c|}{$\mathrm{N} / \mathrm{A}$} & \multicolumn{2}{|c|}{0.7642} \\
\hline \multirow[t]{2}{*}{ Carcinomas $(n=5)$} & $0(0-2)$ & 0.8702 & \multicolumn{2}{|c|}{0.7642} & \multicolumn{2}{|c|}{$\mathrm{N} / \mathrm{A}$} \\
\hline & & Normal & Microadenoma & Macroa & loma & Carcinoma \\
\hline Normal $(n=7)$ & $0(0-2)$ & $\mathrm{N} / \mathrm{A}$ & 0.9541 & & & 0.8706 \\
\hline Microadenoma $(n=11)$ & $0(0-2)$ & 0.9541 & $\mathrm{~N} / \mathrm{A}$ & & & 0.8262 \\
\hline Macroadenoma $(n=30)$ & $0(0-2)$ & 0.455 & 0.3117 & & & 0.648 \\
\hline Carcinoma $(\mathrm{n}=5)$ & $0(0-2)$ & 0.8706 & 0.8262 & & & $\mathrm{~N} / \mathrm{A}$ \\
\hline
\end{tabular}

Median value (range); comparison between groups was performed using with the Kruskal-Wallis test with Conover-Inman correction; GH-oma: GH-secreting adenoma; PRL-oma: Prolactinoma; ACTH-oma: ACTH-secreting adenoma; NFPA: non functioning pituitary adenoma. 


\section{ACTH-secreting adenoma without cytoplasmic staining}

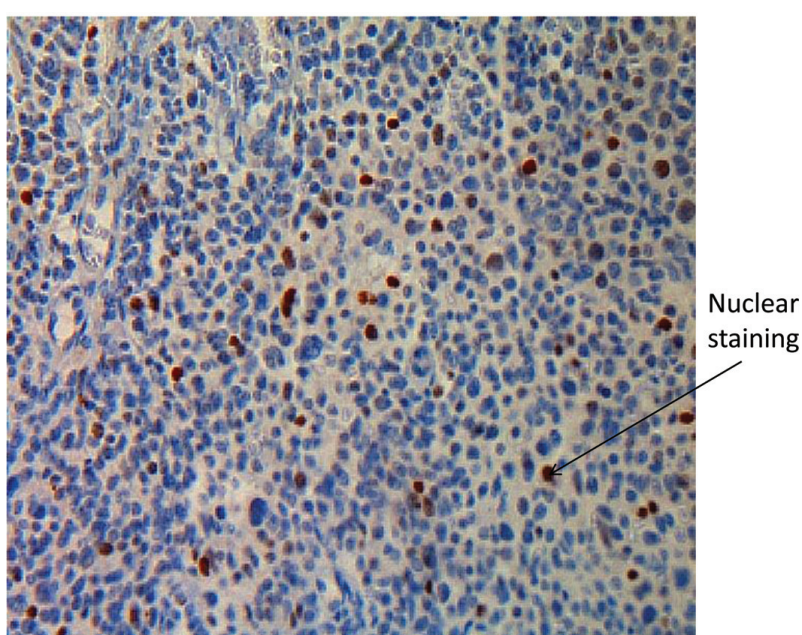

Figure 3. Results for nuclear p21 immunostaining on a pituitary tumour. ACTH-secreting adenoma without cytoplasmic staining (magnification x 400).

statistical significance $(\mathrm{P}=0.06)$. It is of note that one of the cases had received irradiation before the operation.

\section{DISCUSSION}

Our results show that $\beta$-galactosidase, considered as a senescence marker, is over-expressed only in specific subtypes of pituitary adenomas, but is also present in carcinomas considered as a group. Radiotherapy may amplify this process, but there was no evidence for a loss of senescence when comparing ACTH-secreting carcinomas to adenomas, since senescence was not reduced in carcinomas when the irradiated ones were excluded. Finally, changes in p16 and p21 expression suggest that there are tissuespecific pathways involved in senescence activation and that both these proteins play a distinctive and different role.

Since $\beta$-galactosidase was over-expressed only in NFPAs and GH-secreting tumours, it might be suggested that, in the context of senescence, such adenomas behave differently compared to ACTH- and PRL-secreting adenomas. Considering the fact that the great majority of pituitary carcinomas are derived from these latter types of pituitary adenoma, we might speculate that the lack of OIS could allow the occasional PRL- or ACTH-secreting pituitary adenoma

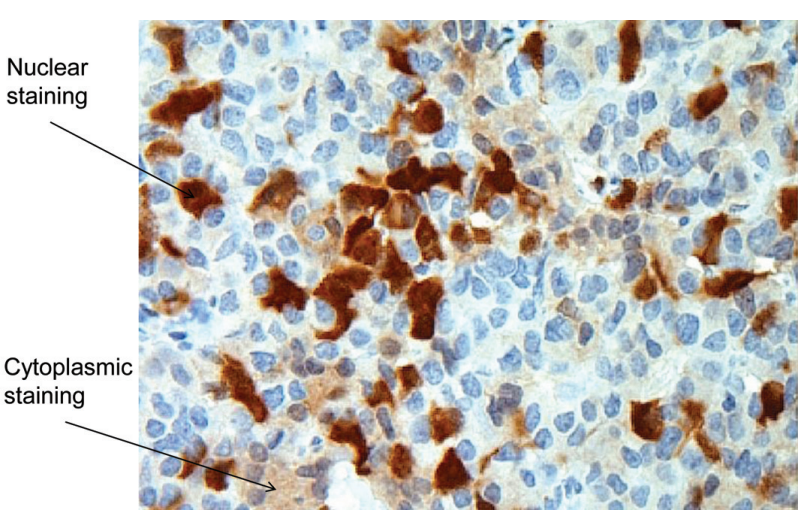

Figure 4A. Examples of nuclear and cytoplasmic p16 immunostaining in pituitary tumours. GH-secreting adenoma (magnification $x$ 1000). Cytoplasmic staining score $=6$. Nuclear staining score: $2.9 \%$ strong + moderate.

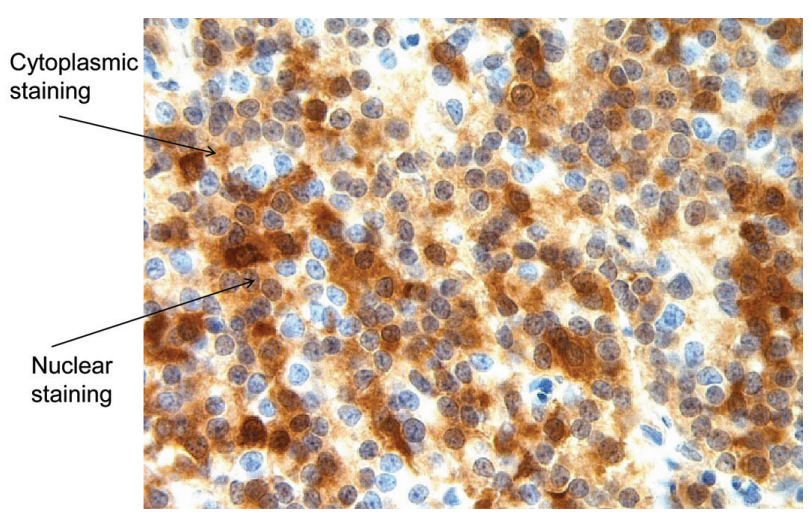

Figure 4B. Examples of nuclear and cytoplasmic p16 immunostaining in pituitary tumours. Non functioning pituitary adenoma (magnification x 1000). Nuclear staining: $2.9 \%$ strong + moderate. Cytoplasmic staining score: 6 .

to develop into a carcinoma. We were only able to investigate a small group of carcinomas, since they are extremely rare. It is of note that pituitary carcinomas too showed apparent activation of senescence even if ineffectively, and indeed their senescence may even have been enhanced by radiotherapy as previously suggested, ${ }^{22}$ implying a protective role for irradiation. It is of interest that both patients with the highest score of $\beta$-galactosidase (scored with 6 ) had been previously irradiated. Hence, excluding both cases from the analysis, pituitary carcinomas no longer differed from either normal pituitary or the overall group of adenomas.

Moreover, the present results imply that senescence 
may not be a specific characteristic of microadenomas but, as opposed to previous suggestions, ${ }^{23}$ this may be more a feature of macroadenomas, underlining an association with the size and the invasiveness of the tumour.

Regarding the mediators of the senescence pathway, the current data support previous findings that the changes in $\mathrm{p} 16$ and $\mathrm{p} 21$ expression between tumour types may possibly be involved in different senescence pathways in these tumours. ${ }^{9}$ Indeed, we noted the presence of a fall in both nuclear and cytoplasmic p16 expression in parallel in all tumours, in accord with previous reports,, 17 and particularly in the carcinomas even after the exclusion of irradiated carcinomas. Falls in cytoplasmic and nuclear p16 were correlated, and the data suggest a transcriptional decrease in p16 in such tumours, which is more pronounced in carcinomas. By contrast, there appeared to be a shift between nuclear and cytoplasmic compartments for $\mathrm{p} 21$, as previously noted, ${ }^{24,25}$ confirmed by their negative association in the correlation analysis, with significant differences between GH-secreting tumours and NFPAs. Hence, we have confirmed previous results of weak cytoplasmic staining for $\mathrm{p} 21,{ }^{14}$ while our results are in agreement with high nuclear staining of p21 in adenomas ${ }^{26}$ and particularly high in $\mathrm{GH}$-secreting adenomas $^{14,26}$ and low in NFPAs. ${ }^{15}$ Thus, while both GH-secreting tumours and NFPAs show similar senescence activation, this was not due to a common change in p16 and p21 expression. The pattern of oncogenic and tumour suppressor gene expression is therefore tumour-type specific for different pituitary tumours. This suggests that even in the presence of OIS, the contextual background may differ according to the specific tumour type and is not a generic uniform process. ${ }^{18,26}$ Thus, changes in $\mathrm{p} 16$ expression are a generic aspect of pituitary tumourigenesis, while p21 shows differential expression in different tumour types, as opposed to previous findings showing both mediators reduced in NFPAs. ${ }^{26,27}$

Overall, the present study does not provide any clear indication as to the process whereby adenomas very rarely transform into carcinomas, although, since such tumours are extremely rare, only small numbers are available for analysis. However, we may say that NFPAs and GH-secreting adenomas rarely result in carcinomas and the presence of senescence may play a crucial role in their more benign behaviour. Ap- parently, there is a different interplay of senescence factors defining benign and malignant tumours. The role of previous treatment such as irradiation should also be considered. Nuclear $\mathrm{p} 21$ was not significantly different in expression compared to the adenomas, in contrast with previous studies of GH-secreting carcinomas where it was reported to be undetectable, ${ }^{14}$ but this was not the case for cytoplasmic p21 which was very weak or negative. On the other hand, both nuclear and cytoplasmic p16 under-expression was more intense in carcinomas compared to that seen in adenomas. The different trends in the expression of both $\mathrm{p} 21$ and $\mathrm{p} 16$ in $\mathrm{ACTH}$ - and prolactin-secreting tumours may predispose them to malignant transformation, but the specific mutational or other changes of malignancy remain unclear. ${ }^{17}$

Finally, we found in all the pituitary adenomas and carcinoma samples a negative correlation between the Ki-67 mitotic index and cytoplasmic staining for both $\mathrm{p} 16$ and $\mathrm{p} 21$. This interesting finding points to the importance of the evaluation of both nuclear and cytoplasmic staining of these important mediators of the senescence process. As nuclear factors are produced in the cytoplasm and transferred to the nucleus, there is always some cytoplasmic staining, which in normal tissues is weak but in tumour tissues might increase, ${ }^{28}$ although its presence is not a general predictor of prognosis. ${ }^{28,29}$ Furthermore, the fact that $\beta$-galactosidase was not correlated with either p16 or p21 may suggest that other mediators play a more central role in this process than the two that we investigated.

In conclusion, we have shown that a marker of senescence such as $\beta$-galactosidase is specifically increased in somatotroph tumours and NFPAs, despite the fact that p21 expression was different between these tumour types and p16 was generally underexpressed in all pituitary tumours; carcinomas appeared to show senescence, but in this small group such tumours showed a similarity to their benign counterparts when irradiated tissue was excluded from the analysis. Considering the small carcinoma group size, no definite conclusions can be derived about the role of senescence in pituitary carcinomas, but we certainly found no evidence for malignant transformation leading to loss of senescence. These results offer further evidence that the oncogenic pathways activated in pituitary tumours are cell-type 
specific, suggesting that senescence may play a role in maintaining the benign nature of certain tumours. The creation of a central registry and in depth investigation of these mediators may shed light on the pathogenesis of these rare tumours.

\section{ACKNOWLEDGMENTS}

Dr K. Alexandraki was awarded a scholarship by the Alexander S. Onassis Public Benefit Foundation. There is no conflict of interest that could be perceived as prejudicing the impartiality of the research reported.

\section{REFERENCES}

1. Fernandez A, Karavitaki N, Wass JA, 2010 Prevalence of pituitary adenomas: a community-based, cross-sectional study in Banbury (Oxfordshire, UK). Clin Endocrinol (Oxf) 72: 377-382.

2. Scheithauer BW, Gaffey TA, Lloyd, et al, 2006 Pathobiology of pituitary adenomas and carcinomas. Neurosurgery 59: 341-353

3. Donangelo I, Melmed S, 2005 Pathophysiology of pituitary adenomas. J Endocrinol Invest 28(11 Suppl International): 100-105.

4. Dworakowska D, Wlodek E, Leontiou CA, et al, 2009 Activation of RAF/MEK/ERK and PI3K/AKT/mTOR pathways in pituitary adenomas and their effects on downstream effectors. Endocr Relat Cancer 16: 1329-1338.

5. Lowe SW, Cepero E, Evan G, 2004 Intrinsic tumour suppression. Nature 432: 307-315.

6. Sherr CJ, 2004 Principles of tumour suppression. Cell 116: 235-246.

7. Kuilman T, Michaloglou C, Vredeveld LC, et al, 2008 Oncogene-induced senescence relayed by an interleukindependent inflammatory network. Cell 133: 1019-1031.

8. Mooi WJ, Peeper DS, 2006 Oncogene-induced cell senescence-halting on the road to cancer. N Engl J Med 355: 1037-1046.

9. Quereda V, Malumbres M, 2009 Cell cycle control of pituitary development and disease. J Mol Endocrinol 42: 75-86.

10. Collado M, Serrano M, 2006 The power and the promise of oncogene-induced senescence markers. Nat Rev Cancer 6: 472-476.

11. Arzt E, Chesnokova V, Stalla GK, Melmed S, 2009 Pituitary adenoma growth: a model for cellular senescence and cytokine action. Cell Cycle 8: 677-678.

12. Going JJ, Stuart RC, Downie M, Fletcher-Monaghan AJ, Keith WN, 2002 'Senescence-associated' betagalactosidase activity in the upper gastrointestinal tract J Pathol 196: 394-400.

13. Itahana K, Campisi J, Dimri GP, 2007 Methods to detect biomarkers of cellular senescence: the senescence- associated beta-galactosidase assay. Methods Mol Biol 371: 21-31.

14. Chesnokova V, Zonis S, Kovacs K, et al, 2008 p21(Cip1) restrains pituitary tumour growth. Proc Natl Acad Sci U S A. 105: 17498-17503.

15. Chesnokova V, Zonis S, Zhou C, et al, 2011 LineageSpecific Restraint of Pituitary Gonadotroph Cell Adenoma Growth. PLoS ONE 6: e17924.

16. Melmed S, 2011 Pathogenesis of pituitary tumours. Nat Rev Endocrinol 7: 257-266.

17. Chesnokova V, Zonis S, Rubinek T, et al, 2007 Senescence Mediates Pituitary Hypoplasia and Restrains Pituitary Tumour Growth. Cancer Res 67: 10564-10572.

18. Machiavelli G, Cotignola J, Danilowicz K, et al, 2008 Expression of p16(INK4A) gene in human pituitary tumours. Pituitary 11: 71-75.

19. Quereda V, Martinalbo J, Dubus P, Carnero A, Malumbres M, 2007 Genetic cooperation between p21Cip1 and INK4 inhibitors in cellular senescence and tumour suppression. Oncogene 26: 7665-7674.

20. Korbonits M, Chahal HS, Kaltsas G, et al, 2002 Expression of phosphorylated p27(Kip1) protein and Jun activation domain-binding protein 1 in human pituitary tumours. J Clin Endocrinol Metab 87: 2635-2643.

21. Norton AJ, Jordan S, Yeomans P, 1994 Brief, high-temperature heat denaturation (pressure cooking): a simple and effective method of antigen retrieval for routinely processed tissues. J Pathol 173: 371-379.

22. Sabin RJ, Anderson RM. Cellular Senescence - its role in cancer and the response to ionizing radiation. Genome Integr 2: 7.

23. Mooi WJ, 2009 Oncogene-induced cellular senescence: causal factor in the growth arrest of pituitary microadenomas? Horm Res 71: Suppl 2: 78-81.

24. Zhou BP, Liao Y, Xia W, Spohn B, Lee MH, Hung MC, 2001 Cytoplasmic localization of p21Cip1/WAF1 by Aktinduced phosphorylation in HER-2/neu-overexpressing cells. Nat Cell Biol 3: 245-252.

25. Weinberg WC, Denning MF, 2002 P21Wafl control of epithelial cell cycle and cell fate. Crit Rev Oral Biol Med 13: 453-464.

26. Neto AG, McCutcheon IE, Vang R, Spencer ML, Zhang W, Fuller GN, 2005 Elevated expression of p21 (WAF1/ Cip1) in hormonally active pituitary adenomas. Ann Diagn Pathol 9: 6-10.

27. Farrell WE, Clayton RN, 2003 Epigenetic change in pituitary tumourigenesis. Endocr Relat Cancer 10: 323-330.

28. Evangelou K, Bramis J, Peros I, et al, 2004 Electron microscopy evidence that cytoplasmic localization of the p16(INK4A) "nuclear" cyclin-dependent kinase inhibitor (CKI) in tumor cells is specific and not an artifact. A study in non-small cell lung carcinomas. Biotech Histochem 79: 5-10.

29. Zhao P, Hu YC, Talbot IC, 2003 Expressing patterns of p16 and CDK4 correlated to prognosis in colorectal carcinoma. World J Gastroenterol 9 : 2202-2206. 